\title{
APROXIMACIÓN SISTÉMICA AL ANÁLISIS DE LA CUENCA DEL RÍO AGÜERA
}

\author{
J. Pozo, A. Elósegui y A. Basaguren \\ Lab. Ecología, Dpto. Biología Vegetal y Ecología, F. Ciencias, \\ Universidad del País Vasco/E.H.U., Apdo. 644. 48080 BILBAO.
}

Keywords: watershed, stream ecosystem, organic matter, structural dynamics

\begin{abstract}
SYSTEM APPROACH TO THE ANALYSIS OF THE AGUERA STREAM WATERSHED

During the last years, the group of Stream Limnology, Laboratory of Ecology, University of the Basque Country, has been studying the Agüera stream watershed from a system approach. This is a medium-sized catchment $\left(145 \mathrm{Km}^{2}\right)$, with diverse lithology and low human density. Main land uses are forestry and livestock.

The Agüera is a moderately mineralized fluvial system, where spatial heterogeneity is disrupted by temporal changes, which are mainly due to sporadic floods. The headwaters receive important allochthonous organic inputs, and this is reflected in the density of shredders and collectors-gatherers. In medium reaches, the water is enriched in nutrients as a result of urban sewage and agricultura1 practices, although the stream shows a high self-purification. With the development of periphyton, scrapers acquire relevance, reflecting the switch from heterotrophy to autotrophy $(\mathrm{P} / \mathrm{R}>1)$. The fluvial community exerts there a stronger control over the streamwater, and the nutrient availability makes the allochthonous organic inputs decay faster.
\end{abstract}

\section{INTRODUCCIÓN}

La impresión que causa la observación de un río, claramente delimitado de su entorno terrestre, contrasta con la dependencia que este tipo de sistemas tiene respecto de lo que le rodea. El estudio de los sistemas fluviales en el marco de su cuenca cobró especial importancia a partir de los años setenta (HYNES, 1975) y, hoy en día, es prácticamente necesario contemplar los procesos que se dan a nivel de la cuenca para abordar una investigación en limnología fluvial.

Muchos de los trabajos realizados durante la última década han partido de los planteamientos recogidos en la teoría del River Continuum Concept (VANNOTE et al., 1980), la cual ha favorecido el avance de la ecología fluvial desde aspectos meramente descriptivos a otros con valor predictivo. La mayoría de estos estudios, y las correspondientes hipótesis planteadas (Nutrient Spiralling, NEWBOLD et al., 1981; Serial Discontinuity Concept, WARD \& STANFORD, 1983...), han sido desarrollados en áreas donde la vegetación de la cuenca estaba prácticamente inalterada.
Hoy en día, la mayoría de las aguas corrientes distan mucho de encontrarse en situación prístina, y aunque los ecosistemas fluviales presentan una notable capacidad de autodepuración, a menudo ésta se ve sobrepasada, por lo que los procesos que ocurren en los cauces están condicionados por las modificaciones que introduce el hombre a nivel de toda la cuenca. Se generan así perturbaciones en los ríos que desplazan el equilibrio de los procesos naturales de modo más o menos intenso y más o menos persistente, y que pueden ser evaluadas mediante el análisis integral de cuencas fluviales (HORNBECK \& SWANK, 1992).

Si bien en la Península Ibérica hay publicados estudios intensivos a nivel de cuenca (SABATER et al., 1990; POZO et al., 1994a), es indudable que queda mucho por desarrollar en este sentido, máxime si tenemos en cuenta la diversidad de nuestros sistemas fluviales. En concreto, hay que hacer hincapié en la importancia de la variabilidad de los ríos de distintas zonas geográficas a escalas espaciales y temporales complementarias (GORE \& MILNER, 1990), y en la incidencia de dicha variabilidad sobre las características y funcionamiento de las comunidades 
fluviales (WARD, 1992).

Previo al estudio de sistemas profundamente humanizados, el análisis integral de cuencas fluviales debe partir del estudio de sistemas poco modificados, de manera que pueda discernirse la dinámica natural de los ríos de los cambios asociados a las perturbaciones.

En este sentido, el equipo de Limnología Fluvial del Laboratorio de Ecología de la Universidad del País Vasco/EHU viene investigando la cuenca del río Agüera desde 1988, gracias a varios proyectos subvencionados por distintas entidades públicas. Se está adquiriendo un conocimiento preciso de la misma, que permitirá a medio plazo extrapolar la información obtenida a cuencas cercanas. Hasta ahora, se ha efectuado una caracterización de la cuenca, un análisis detallado de las variaciones espaciotemporales en la físico-química del agua, de la comunidad de macroinvertebrados bentónicos, de la biomasa perifítica, y de los procesos de transporte y descomposición de materia orgánica, así como un inicio al estudio del metabolismo fluvial. Todo ello hace que la cuenca del Agüera sea la mejor conocida del País Vasco desde el punto de vista limnológico.

\section{ÁREA DE ESTUDIO}

La cuenca del río Agüera (Fig. 1), situada entre la región oeste de Vizcaya y la parte oriental de Cantabria, en el norte de la Peninsula Ibérica, ocupa una extensión de $145 \mathrm{Km}^{2}$, siendo de forma estrecha y alargada. El drenaje más importante de los $175 \mathrm{Km}$ que constituyen la red hidrográfica comprende tramos de orden 1 (STRAHLER, 1986), unos $106 \mathrm{Km}$ de cauces constituídos por arroyos de corto recorrido. Desde el punto de vista geológico, se asienta sobre substrato de origen cretácico, de litología silícea salvo en una amplia banda calcárea central, constituyendo una zona con abundantes fallas y plegamientos.

La cuenca está caracterizada por su escasa densidad de población $\left(20 \mathrm{hab} / \mathrm{Km}^{2}\right)$ y por una dedicación fundamentalmente agrícola y forestal. Se conservan superficies relativamente importantes de bosques autóctonos (robledal y encinar) pero más del $25 \%$ del territorio se destina a repoblaciones de Pinus radiata o Eucalyptus globulus, además de un $20 \%$ de prados y cultivos, cuya producción se orienta a la ganadería vacuna. La prácticamente nula actividad industrial hace que los vertidos urbanos y los cambios de usos del suelo se puedan considerar como los principales factores de perturbación de los sistemas fluviales.

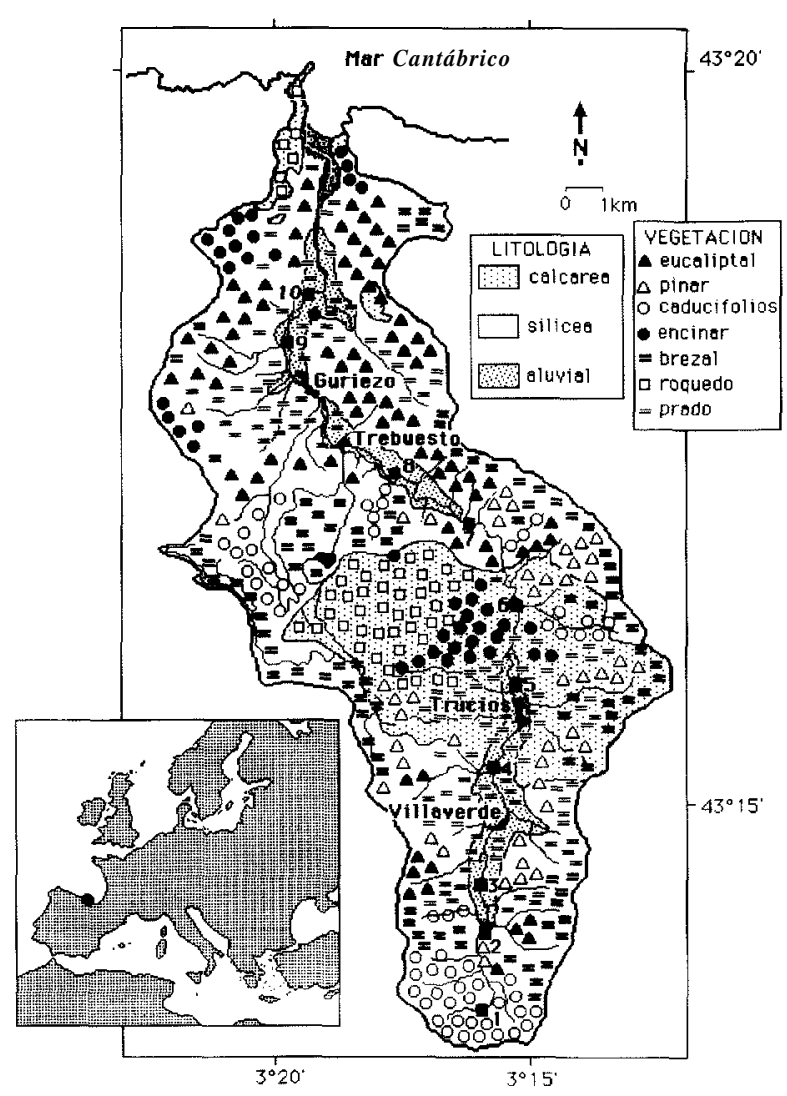

Figura 1.- Area de estudio: características geológicas, usos del suelo, y localización de los principales núcleos urbanos. Los números 1 a 10 hacen referencia a las estaciones de muestreo del cauce principal mencionadas en el texto.

Figure 1.- Study site: geological features, soil uses and location of the main villages. Numbers 1 to 10 are the sampling stations in the main channel mentioned in the text.

\section{CARACTERIZACIÓN FÍSICO-QUÍMICA DE LOS CAUCES}

Para el estudio de las características físico-químicas del río Agüera se establecieron diez estaciones de muestreo, desde la cabecera hasta la desembocadura, que se visitaron quincenalmente durante dos periodos (Noviembre 88-Mayo 89 y Diciembre 89-Enero 91). Cincuenta y dos puntos adicionales, en el conjunto de afluentes, fueron visitadas con una periodicidad estacional durante un año, a fin de estudiar la relación entre el uso del suelo y las características de las aguas. Seis estaciones, inmediatamente aguas arriba y abajo de los principales núcleos urbanos, permitían evaluar el efecto de estos sobre el cauce fluvial.

Desde el punto de vista físico-químico, las aguas del río Agüera son, en general, de buena calidad, debido al bajo 
nivel de actividades humanas y al buen estado de conservación de la cuenca (ELÓSEGUI \& POZO, 1992; ELÓSEGUI \& POZO, en prensa). La figura 2 muestra las variaciones espaciales y temporales en la conductividad, y la 3 las del fosfato, ejemplos de variables conservativa y no conservativa, respectivamente. La conductividad presenta gradientes espaciales acusados, como consecuencia de los cambios en la composición litológica del sustrato, siendo las diferencias temporales achacables casi en su totalidad a las variaciones del caudal. El fosfato, asociado a los núcleos urbanos, aumenta sus concentraciones en las estaciones 4, 5 y 9, evidenciándose una disminución notable de su concentración aguas abajo de la estación 5. Las mayores diferencias observadas, pues, se producen por cambios en la geología de las cuencas que drenan los diversos afluentes que se incorporan sucesivamente al eje principal, y por influencia de asentamientos humanos (GONZÁLEZ et al., 1993, 1994).

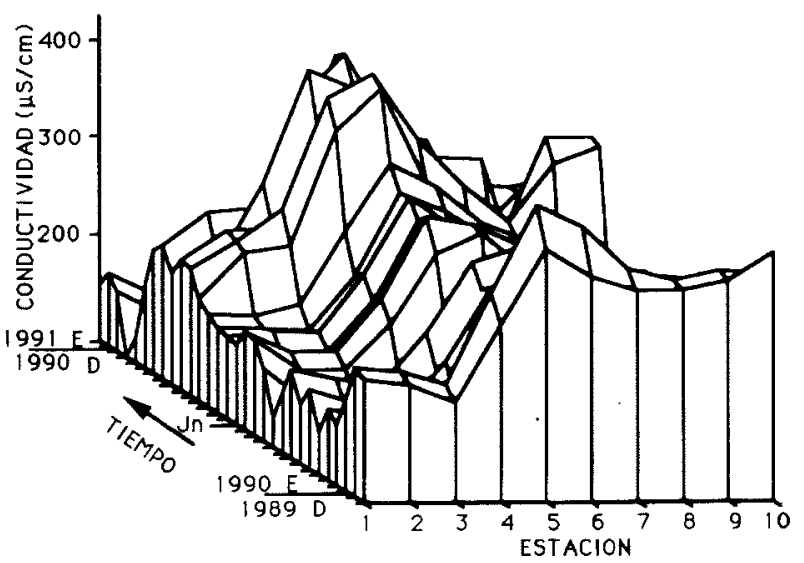

FIGURA 2.- Variaciones espacio-temporales de la conductividad en el eje principal del río Agüera.

FIGURE 2.- Spatio-temporal variations in conductivity along the main channel of the Agüera stream.

Las características de las aguas varían bastante a lo largo del año, principalmente en función del régimen hidrológico, sin que haya un ciclo estacional propio en la mayor parte de las variables determinadas (ELÓSEGUI \& POZO, en prensa). En general, a descargas elevadas, se produce una dilución y homogeneización de las aguas del eje principal, y disminuye la importancia del control biótico sobre las características de las mismas. En épocas de baja precipitación aumentan los efectos locales, tanto de la contaminación como de otras fuentes de variabilidad, observándose una gran capacidad de regulación por parte de la comunidad fluvial, que conlleva tasas de autodepura-

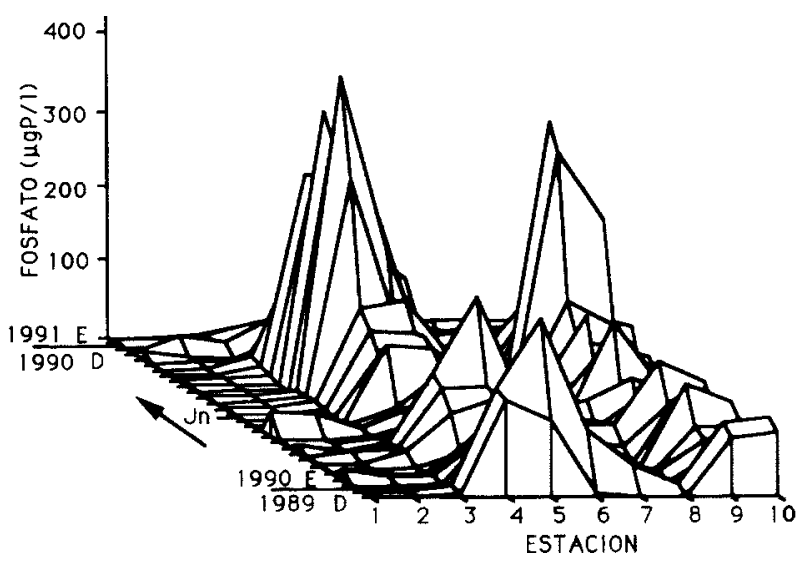

FIGURA 3.- Variaciones espacio-temporales de la concentración de fosfato en el eje principal del río Agüera.

FIGURE 3.- Spatio-temporal variations in phosphate concentration along the main channel of the Agüera stream.

ción elevadas en tramos medios (ELÓSEGUI et al., en prensa).

Un análisis de componentes principales sobre los datos de las variables físico-químicas obtenidas durante un ciclo anual (Fig. 4) destaca en primer lugar la mineralización, y en segundo la eutrofización, como factores que afectan al quimismo de las aguas. La heterogeneidad espacial es destruída por cambios temporales asociados al régimen hidrológico sin una componente estacional clara (ELÓSEGUI \& POZO, en prensa). El gradiente longitudinal de aumento de mineralización, general para la mayoría de los ríos (MARGALEF, 1983), aparece deformado, mostrando discontinuidades por cambios en la litología y en los aportes urbanos. Así, las estaciones de cabecera, desde la 1 a la 3, aparecen agrupadas como zonas poco mineralizadas y no eutrofizadas (Fig. 4); el río aumenta su carga de sales y nutrientes a su paso por los núcleos de Villaverde y Trucíos (estaciones 4 y 5); posteriormente, hay una zona de autodepuración (estaciones 6 a 8 ) en la que disminuye la conductividad y, sobre todo, la eutrofia; en el tramo final, al atravesar Guriezo (estación 9), se vuelve a cargar de nutrientes. En último término se constata la dependencia que tiene la composición de las aguas de la organización espacial de la cuenca (SABATER et al., 1990; FERNÁNDEZ ALÁEZ et al., 1992; ELÓSEGUI \& POZO, 1992; POZO et al., 1994a).

\section{RECURSOS ENERGÉTICOS}

Como estima de la potencialidad autótrofa del río 

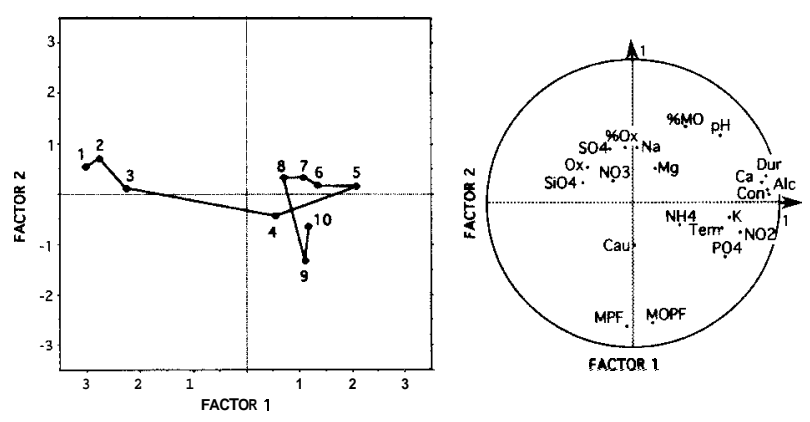

FIGURA 4.- Resultados del ACP realizado sobre datos obtenidos durante un ciclo anual en las estaciones 1 a 10. Localización media de cada estación en el plano factorial 1-2 (izquierda) y correlaciones de las variables con los dos primeros factores (derecha).

FIGURE 4.- Results of the PCA performed on data obtained from an annual cycle at stations 1 to 10 . Average location of each station on the factorial plane 1-2 (left) and correlations between variables and factors 1 and 2 (right).



FIGURA 5.- Variaciones espacio-temporales de la bioniasa perifítica $\left(\mathrm{g} / \mathrm{m}^{2}\right)$ en el eje principal del río Agüera.

FIGURE 5.- Spatio-temporal variations in periphytic biomass $\left(\mathrm{g} / \mathrm{m}^{2}\right)$ along the main channel of the Agüera stream

Agüera, se estudió la biomasa perifítica durante un año (Enero 1990 a Enero 1991) y con una periodicidad mensual, en cinco de las diez estaciones del cauce principal, mediante técnicas gravimétricas (biomasa) y espectrofotométricas (clorofila a). La densidad de perifiton (Fig. 5) va aumentando desde las estaciones de cabecera hasta los tramos medios del río, disminuyendo en los tramos finales. Ese modelo refleja, en parte, las previsiones teóricas del RCC (VANNOTE et al., 1980), pero los máximos encontrados a nivel medio del eje principal no son una consecuencia del tamaño que va adquiriendo el río, sino de la modificación de los usos del suelo de la cuenca por eliminación de la vegetación riparia. Esto genera una disminución de la sombra e incrementos de temperatura y luz, que favorecen el crecimiento algal y pueden convertir los cursos heterotróficos en autotróficos (HOLOPAINEN et a 1 , 1991) A ello habría que sumar el efecto causado por el aumento de la concentración de nutrientes provenientes de los vertidos urbanos aguas arriba El desarrollo algal en los tramos medios del río Aguera no sigue un modelo estacional claro, sino que su dinámica responde a los cambios del régimen hidrológico. Crecidas de caudal en Abril de 1990 y durante el otoño-invierno siguiente (POZO et al., 1994a) hacen disminuir los valores y homogenizan el río, mientras que en situaciones de estabilidad hidrológica basal se adquieren elevadas biomasas (Fig. 5). En cabecera, sólo durante el final del verano y principios de otoño se alcanzan biomasas perifíticas significativas.

Dada la segregación espacial en la manifestación de los productores primarios del bentos, muy a menudo la productividad de los ecosistemas fluviales de pequeño orden está fundamentada en el volumen de aportes alóctonos de materia orgánica, en su mayor parte procedentes de la vegetación riparia, que se introducen principalmente en la cabecera y vía tributarios. El régimen hidrológico, la morfología y la zonación de la vegetación de la cuenca, junto a otros procesos de naturaleza biótica, habitualmente son considerados como determinantes de la cantidad y valor cualitativo de la materia orgánica acumulada en el cauce y aledaños del río (BARNES \& MINSHALL, 1983; CUMMINS et al., 1989; GOLLADAY et al., 1989; MINSHALL et al., 1992).

La disponibilidad de materia orgánica particulada gruesa (MOPG) de origen alóctono en el río Agüera se manifiesta claramente dependiente del tramo de río, tanto por el tipo de vegetación riparia como por la importancia decreciente que va teniendo la misma con el ensanchamiento del cauce (POZO et al., 1994b). Además de este efecto, el aumento de tamaño del canal en los ríos tiende a incrementar la importancia relativa de las entradas provenientes de aguas arriba (VANNOTE et al., 1980; CUMMINS, 1988). Por otra parte, la estacionalidad de los aportes y el régimen hidrólogico, sobre todo por efecto de las crecidas, regulan la cantidad de materia disponible en el lecho del río a lo largo del año (Tabla 1). Por lo que se refiere al transporte de MOPG, los resultados disponibles sobre este río (POZO et al., 1994b) no muestran una diferenciación espacial clara. El modelo de variación anual de la materia orgánica particulada gruesa concuerda con otros estudios 
que muestran máximos en otoño o durante las crecidas (LIKENS et al., 1985). Este recurso constituye una muy pequeña fracción de la materia orgánica particulada total (Fig. 6), como también señalan CELLOT et al. (1991); esto sugiere que la MOPG que ingresa en el río es transportada sólo cortas distancias y es procesada cerca del lugar de entrada (HALL, 1972).

TABLA 1.- Rango de valores medios de materia orgánica particulada gruesa sedimentada (g PSLC/m2) obtenidos en una serie de muestreos realizados entre Noviembre de 1988 y Febrero de 1991 en el no Agüera en una estación de cabecera (1) y en otra de tramo medio (7). Entre paréntesis se indica el error stándar en el muestreo correspondiente.

TABLE 1.- Range of mid values of benthic particulate organic matter (g AFDW/m2) obtained in a set of samplings between November 1988 and February 1991 in the Agüera stream at two sites (1, headwater; 7, mean reach). Standard error of the corresponding sampling is shown between brackets

\begin{tabular}{lcc}
\hline & MAXIMO & MINIMO \\
ESTACION 1 & 217,3 & 8,7 \\
& $(56,3)$ & $(4,3)$ \\
ESTACION 7 & 22,5 & 2,8 \\
& $(8,0)$ & $(0,8)$ \\
\hline
\end{tabular}

\section{MACROINVERTEBRADOS BENTÓNICOS}

Una aproximación al estudio de estos organismos desde un punto de vista funcional consiste en clasificarlos según su forma de alimentación (MERRIT \& CUMMINS, 1978).

La diferente composición funcional que presentan las comunidades de macroinvertebrados a lo largo del río (Fig. 7) es resultado de las exigencias ecológicas de los organismos, que responden al gradiente longitudinal de variación que se establece en los cursos de agua, y está relacionado con la condición heterotrofia-autotrofia de los ríos señalada por VANNOTE et al. (1980). Los fragmentadores, que utilizan la energía procedente de los ecosistemas terrestres en forma de hojarasca, dominan junto con los recolectores en el tramo superior del Agüera y pierden su preponderancia en las zonas humanizadas y con la eliminación de la vegetación arbórea riparia (estaciones $\mathbf{3}$ a $5)$, donde filtradores y generalistas aumentan. Con la disminución de la presión humana y el ensanchamiento del cauce, los recolectores, junto con los raspadores y la recuperación de los fragmentadores producen un nuevo cambio en la comunidad de macroinvertebrados (estaciones 6 a 8). En los tramos bajos, con la intensificación de los vertidos urbanos, los filtradores dominan la biomasa

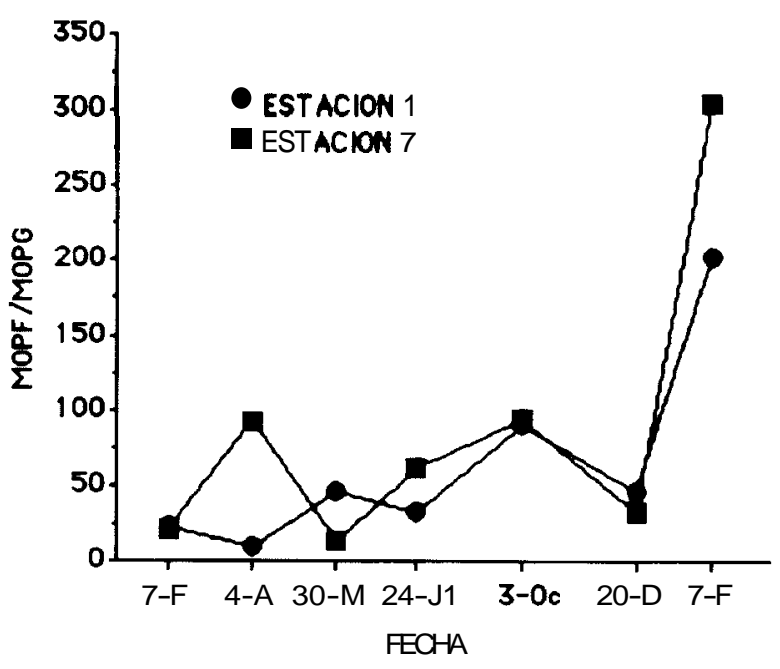

FIGURA 6.- variaciones temporales en la relación materia orgánica particulada fina (MOPF)/materia orgánica particulada gruesa (MOPG) del agua en las estaciones 1 y 7 del río Agüera (Febrero 1990-Febrero 1991). FIGURE 6.- Temporal variation of the FPOM/CPOM ratio in the water at sites 1 and 7 of the Agüera stream (February 1990-February 1991).

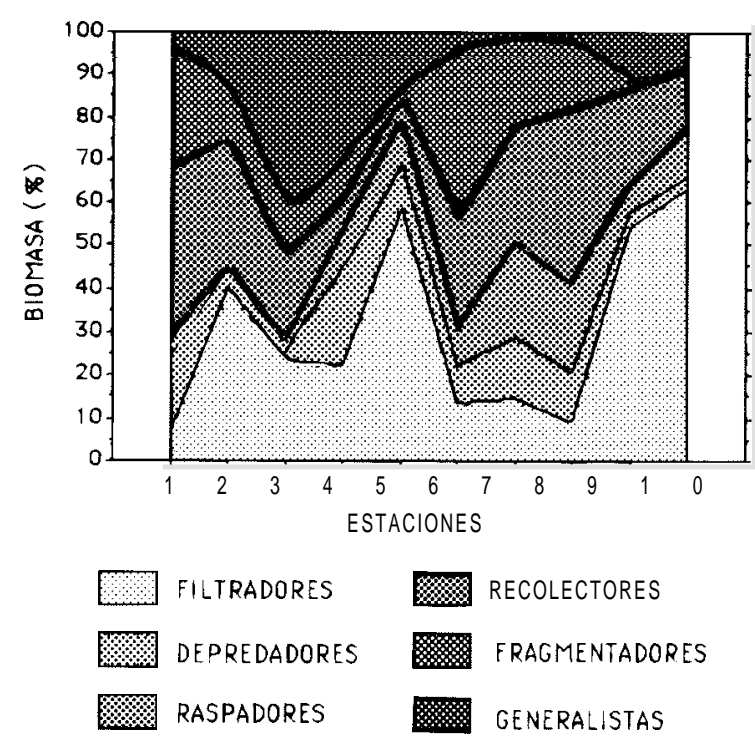

FIGURA 7: Variaciones espaciales en la estructura funcional de la comunidad de macroinvertebrados en el eje principal del río Agüera durante el otoño de 1988.

FIGURE 7: Spatial variations of the functional structure of the macroinvertebrate community in the main channel of the Agüera stream during autumn, 1988.

de macroinvertebrados. Obviamente, el esquema presentado no es estático. Desde una perspectiva temporal, los cambios más significativos en la abundancia y estructura de las comunidades bentónicas ocurren a nivel de los tra- 
mos medios, y están controladas por factores como fuentes de alimento, ciclos vitales y régimen hidrológico (RIAÑO et al., 1993; BASAGUREN et al., en prep.). Por una parte, se observa una estrecha relación entre desarrollo de productores primarios y abundancia de raspadores; por otra, es posible encontrar relaciones significativas entre densidad de fragmentadores y disponibilidad de MOPG.

\section{METABOLISMO FLUVIAL}

Estimamos el metabolismo de las estaciones 1, 5 y 7 en cuatro épocas del año, a partir de las variaciones nictemerales en la concentración de oxígeno, siguiendo la metodología propuesta por THYSSEN \& KELLY (1985). Estos resultados permiten asignar a cada tramo un nivel dentro del gradiente autotrofia-heterotrofia. La cabecera (Fig. 8) muestra muy pocos cambios durante el día, siendo la influencia de los productores muy escasa. Las estaciones inferiores, por su parte, manifiestan variaciones importantes a esta escala temporal, reflejadas principalmente en la saturación de oxígeno, o en otras variables como pH o nivel de nutrientes (ELÓSEGUI \& POZO, en prep.), como consecuencia del ritmo diario de producción de la comunidad, al que se solapa el ritmo de vertidos urbanos, especialmente en la estación 5 (GONZÁLEZ et al., 1993).

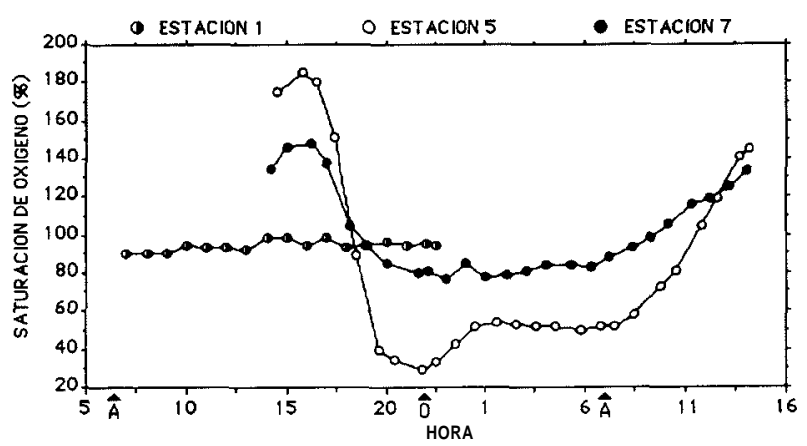

FIGURA 8.- Variación nictemeral de la saturación de oxígeno en las estaciones 1, 5 y 7, en Julio de 1990. A y O marcan la hora del alba y del ocaso, respectivamente.

FIGURE 8.- Diel vanation of the oxygen saturation at sites 1, 5 and 7, July 1990. A and O are dawn and dusk, respectively.

La cabecera es claramente heterotrófica $(\mathrm{P} / \mathrm{R}<1$, datos no publicados), aumentando este cociente aguas abajo, como ocurre en otros ríos (BENKE et al., 1988). La producción primaria de tramos medios, que puede ser importante durante todo el año 'si se dan las condiciones de estabilidad hidrológica necesarias, aumenta hacia el verano, aun- que el incremento de la respiración, asociado al aumento de la temperatura del agua, puede determinar cocientes $\mathrm{P} / \mathrm{R}$ inferiores a 1 , como suele ocurrir en estaciones de mayor nivel de eutrofia (QUINN \& Mc FARLANE, 1989). La variabilidad temporal del cociente $\mathrm{P} / \mathrm{R}$ ha sido citada por numerosos autores (ej. MINSHALL et al., 1992), pero los patrones estacionales pueden diferir mucho entre los distintos sistemas fluviales. A escala de observación nictemeral, pues, podemos deducir la importancia que tiene la comunidad fluvial del río Agüera en el procesado de los vertidos, y en el control de las características físico-químicas del agua.

La descomposición del material orgánico particulado es un proceso igualmente ligado a los cambios que tienen lugar a lo largo del río. Al estudiar las dinámicas del procesado de las hojarascas de aliso (Alnus glutinosa), especie habitual en la mayoría de los cauces de esta cuenca, y de eucalipto (Eucalyptus globulus), extraña en determinadas zonas, aparecían diferencias espaciales (POZO, 1993). En estaciones de cabecera, donde el eucalipto está ausente y las aguas son pobres en nutrientes, la hojarasca de eucalipto se descomponía más lentamente que la del aliso. Aguas abajo, en zonas con repoblaciones de eucaliptos y habiéndose recibido aportes de nutrientes. las diferencias entre ambas hojas desaparecían. El procesado de un mismo tipo de hojarasca puede sufrir importantes variaciones interanuales en un mismo lugar (Fig. 9), probablemente relacionado con el régimen hidrológico, condicionante de las tasas de metabolismo fluvial; de hecho, las mayores pérdidas de peso ocurrieron en la época de prolongada estabilidad hidrológica (experimentos de 1988). La actividad microbiana genera un enriquecimiento en fósforo del material, en función del tramo de río (Tabla 2), lo cual parece condicionar la dinámica de la descomposición, especialmente en hojas de baja calidad como las de eucalipto. El fósforo juega un papel clave en la comunidad fluvial, que muestra una gran capacidad de toma del mismo, contribuyendo a la autodepuración del río Agüera (ver figura 3). Sobre todo, por debajo de Trucíos, las condiciones oligotróficas se restauran en un tramo breve; la eficiencia de la autodepuración aumenta con la biomasa algal, aunque la relación no es sencilla (VYMAZAL, 1988).

Otros nutrientes inorgánicos, además del fosfato, están sometidos igualmente a la retención. La zona más retentiva es el tramo 5-8, por su gran actividad metabólica. En una serie de 26 muestreos realizados en este tramo a lo largo del año, se detectó retención de silicato en 13 ocasiones, con una disminución media del $61 \%$ de la carga 


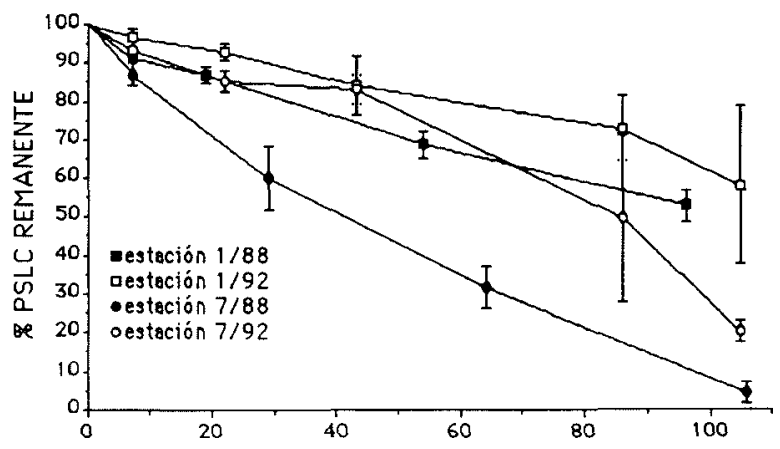

Figura 9: Dinámica de la descomposición de la hojarasca de eucalipto en las estaciones 1 y 7 del río Agüera en experimentos llevados a cabo en 1988 y 1992.

Figure 9: Dynamics of eucalyptus litter processing at sites 1 and 7 of the Aguiera stream; experiments carried out in 1988 and 1992.

TABLA 2.- Concentración media de fósforo en la hojarasca de aliso y eucalipto, antes de comenzar la incubación en el río y al cabo del tiempo en que se hace máxima. Entre paréntesis se indica el error stándar. TABLE 2.- Mean concentration of phosphorus in litter of alder and eucalyptus at the start of the experiments and after it become maximun. Standard error is shown between brackets.

\begin{tabular}{|c|c|c|c|c|c|}
\hline \multirow[t]{2}{*}{ ESPECIE } & \multirow[t]{2}{*}{ INICIAL } & \multicolumn{4}{|c|}{ MAXIMAALCANZADA } \\
\hline & & Estación A & Estación 1 & Estación 7 & Estacióng \\
\hline ALISO & $\begin{array}{r}0,044 \\
(0,006) \\
\\
\text { DIAS }\end{array}$ & $\begin{array}{c}0.046 \\
(0.003) \\
106\end{array}$ & $\begin{array}{l}0,036 \\
(0.004) \\
7\end{array}$ & $\begin{array}{l}0.084 \\
(0,006) \\
64\end{array}$ & $\begin{array}{c}0.107 \\
(0014) \\
64\end{array}$ \\
\hline EUCALIPTO & $\begin{array}{r}0,038 \\
(0,004) \\
\\
\text { DIAS }\end{array}$ & $\begin{array}{l}0.040 \\
(0.006) \\
29\end{array}$ & $\begin{array}{l}0,033 \\
(0,010) \\
29\end{array}$ & $\begin{array}{c}0.073 \\
(0,006) \\
64\end{array}$ & $\begin{array}{c}0,105 \\
(0004) \\
64\end{array}$ \\
\hline
\end{tabular}

transportada, retención de nitrito en 19 ocasiones, con una disminución media del $65 \%$ de la carga, y disminución de amonio en 15 ocasiones, con una media del 68\% (ELÓSEGUI et al., en prensa). La retención de nitrato no es evidente en muchos casos, posiblemente como resultado de los niveles relativamente altos de este ión en las aguas de la cuenca. Las zonas donde se producen más aportes son el tramo 3-4 y el 8-10, coincidentes con Villaverde y Guriezo, respectivamente.

\section{SÍNTESIS Y PERSPECTIVA}

Los resultados obtenidos hasta ahora nos muestran el río Agüera como un sistema complejo donde la comunidad responde al mosaico de condiciones ambientales producido por los cambios en morfometría, litología, usos del suelo, vertidos, alteraciones del cauce. El río muestra una gran capacidad de recuperación frente a las actividades humanas, cuya mayor influencia se manifiesta a nivel de los núcleos urbanos. Las crecidas, de carácter impredecible. son el principal factor de perturbación, alterando los patrones espaciales de las variables abióticas y produciendo el arrastre y redistribución del bentos. La comunidad fluvial muestra un alto grado de elasticidad ante este tipo de eventos.

A partir de este esbozo de funcionamiento del ecosistema, quedan abiertos amplios campos de investigación: estrategias alimentarias dentro de la comunidad de macroinvertebrados, que permitan explicar su papel funcional; establecimiento de relaciones entre la vegetación de la cuenca y el balance orgánico del río; metabolismo fluvial y capacidad de autodepuración, etc.

Es necesario recalcar la necesidad de conjuntar estudios de procesos globales de funcionamiento del ecosistema con otros más detallados sobre aspectos particulares, que den información precisa sobre algunos elementos clave de tales procesos. El análisis integral de cuencas fluviales se muestra como una herramienta útil para sentar las bases científicas necesarias en la planificación del territorio para múltiples usos (HORNBECK \& SWANK, 1992). A partir de ahí, podría operar el cambio hacia un punto de vista preventivo (PERRY, 1994) en la gestión y conservación de los ecosistemas fluviales.

\section{AGRADECIMIENTOS}

Este trabajo es una contribución a partir de los resultados obtenidos en diversos proyectos financiados (UPV 118.310-0067188, PIGV 8924, UPV 118.310-EA154192 у DGICYT PB 459/92). Nuestro agradecimiento a los que han colaborado con nosotros en el desarrollo de esos proyectos: X. Arana, Z. Arteaga, T. Bargos, J.R. Díez, M. Escobal, I. García, E. González, J.M. González, M. Herrera, J. Molinero, M. Onaindía, P. Riaño, A. Rubio, E. Ruiz, y A. Uriarte.

\section{BIBLIOGRAFÍA}

BARNES, J.R. \& MINSHALL, G.W., 1983. Stream ecology: An historical and current perspective. In : J.R. BARNES \& G.W. MINSHALL (Editors), Stream Ecology. Application and testing of general ecological theory. Plenum, New York, pp: 1-5.

BENKE, A.C., HALL, C.A.S., HAWKINS, C.P., LOWE-MCCONNELL, R.H., STANFORD, J.A., SUBERKROPP, K. \& WARD, J.V., 1988. Bioenergetic considerations in the analysis of stream ecosystems. J. N. Am. 
Benthol. Soc., 7: 480-502.

CELLOT, B., BERLY, A., TACHET, H. \& BOURNAUD, M., 1991. Composition and dynamics of suspended matter in the Upper Rhone, upstream from Lyon. Verh. Internat. Verein. Theor. Limnol., 24: 1789-1794.

CUMMINS, K.W., 1988. The study of stream ecosystems. BioScience, 24: 631-641.

CUMMINS, K.W., WILZBACH, M.A., GATES, D.M., PERRY, J.B \& TALIAFERRO, W.B., 1989. Shredders and riparian vegetation. BioScience, 39: 24-30.

ELÓSEGUI, A. \& POZO, J., 1992. Physico-chemical characteristics of the Agüera River (Spain) during an unusual hydrologically period. Annls. Limnol., 28: 85-96.

ELÓSEGUI, A. \& POZO, J., en prensa. Spatial versus temporal variability in the physico-chemical characteristics of the Agüera stream (North Spain). Acta Oecol.

ELÓSEGUI, A., ARANA, X., BASAGUREN, A. \& PO$\mathrm{ZO}$, J., en prensa. Self-purification processes in a medium-sized stream. Environ. Man.

FERNÁNDEZ-ALÁEZ, M., FERNÁNDEZ-ALÁEZ, C. \& CALABUIG, E.L., 1992. The Esla river basin: from the Cantabrian mountain to the Duero. In: C. MONTES, C. DUARTE \& J. GARCÍA-AVILÉS (Editors), Limnology in Spain. Asociación Española de Limnología. Granada, pp: $131-140$.

GOLLADAY, S.W., WEBSTER, J.R. \& BENFIELD, E.F., 1989. Changes in stream benthic organic matter following watershed disturbance. Holarc. Ecol., 12: 96-105.

GONZÁLEZ, E., ELÓSEGUI, A. \& POZO, J., 1993. Influencia de los núcleos urbanos en la variabilidad físicoquímica del río Agüera. Kohie 21: 5-15.

GONZÁlEZ, E., ELósEGUI, A. \& POZO, J., 1994. Changes in the physico-chemical characteristics of the Agüera streamwater associated with human settlements. Verh. Internat. Verein. Theor. Limnol. 25: 1733-1738

GORE, J.A. \& MILNER, A.M., 1990. Island biogeographical theory: can it be used to predict lotic recovery rates? Environ. Man., 14: 737-753.

HALL, C.A.S., 1972. Migration and metabolism in a temperate stream ecosystem. Ecology, 53: 585-604.

HOLOPAINEN, A.L., HUTtUNEN, P. \& AHTIAINEN, M., 1991. Effects of forestry practices on water quality and primary productivity in small forest brooks. Verh. Internat. Verein. Theor. Limnol., 24: 1760-1766.

HORNBECK, J.W. \& SWANK, W.T., 1992. Watershed ecosystem analysis as a basis for multiple-use management of eastern forests. Ecol. Appl., 2: 238-247.

HYNES, H.B.N., 1975. Edgardo Baldi memorial lectu- re. The stream and its valley. Verh. Internat. Verein. Theor. Limnol., 19: 1-15.

KELLY, M.G., THYSSEN, N. \& MOESLUND, B., 1983. Light and the annual variation of oxygen- and carbon-based measurements of productivity in a macrophytedominated river. Limnol. Oceanogr., 28: 503-515.

LIKENS, G.E., BORMANN, F.H., PIERCE, R.S. \& EATON, J.S., 1985. The Hubbard Brook Valley. In: G.E. LIKENS (Editor), An ecosystem approach to aquatic ecology. Mirror lake and its environment. Springer Verlag, New York, pp. 9-39.

MARGALEF, R., 1983. Limnología. Omega, Barcelona, $1010 \mathrm{pp}$.

MERRITT, R.W. \& CUMMINS, K.W., 1978. An introduction to the aquatic insects of North America. Kendall/Hunt, Dubuque, $441 \mathrm{pp}$.

MiNSHALl, G.W., PETERSEN, R.C., BOTT, T.L., CUSHING, C.E., CUMMINS, K.W., VANNOTE, R.L. \& SEDELL, J.R., 1992. Stream ecosystem dynamics of the Salmon River, Idaho: an 8th-order system. J. N. Am. Benthol. Soc., 11: 111-137.

NEWBOLD, J.D., ELWOOD, J.W. \& O'NEILL, R.V., 1981. Measuring nutrient spiralling in streams. Can. J. Fish. Aquat. Sci., 38: 860-863.

PERRY, J.A., 1994. Water quality in the 21 st. century: proactive management at the ecosystem level. Limnética 10(1): 5-13.

POZO, J., 1993. Leaf litter processing of alder and eucalyptus in the Aguiera stream system (North Spain). I. Chemical changes. Arch. Hydrobiol., 127: 299-317.

POZO, J., ELÓSEGUI, A. \& BASAGUREN, A., 1994a. Seston transport variability at different spatial and temporal scales in the Agüera watershed (North Spain). Wat. Res., 28: 125-136.

PozO, J., BASAguren, A. \& Elósegui, A., 1994b. Transported and benthic coarse particulate organic matter in the Agüera stream. Verh. Internat. Verein. Theor. Limnol. 25: 1723-1726.

QUINN, J.M. \& MCFARLANE, P.N., 1989. Effects of slaughterhouse and dairy factory wastewaters on epilithon: a comparison in laboratory streams. Wat. Res., 23: 1267-1273.

RIAÑO, P., BASAGUREN, A. \& POZO, J., 1993. Variaciones espacio-temporales de las comunidades de macroinvertebrados bentónicos del río Agüera (País VascoCantabria). Limnética. 9: 19-28.

SABATER, F., SABATER, S. \& ARMENGOL, J., 1990. Chemical characteristics of a Mediteranean river as 
influenced by land uses in the watershed. Wat. Res., 24: 143-155.

STRAHLER, A.N., 1986. Geografía física. Omega. Barcelona, $767 \mathrm{pp}$.

THYSSEN, N. \& KELLY, M.G., 1985. Water-air exchange of carbon dioxide and oxygen in a river: measurement ünd comparison of rates. Arch. Hydrohiol., 105: 219228.

VANNOTE, R.L., MINSHALL, G.W., CUMMINS, K.W., SEDELL, J.R. \& CUSHING, C.E., 1980. The River Continuum Concept. Can. J. Fish. Aquat. Sci., 37: 130137.
VYMAZAL, J., 1988. The use of periphyton communities for nutrient removal from polluted streams. Hydrobiologia, 166: 225-237.

WARD, J.V., 1992. Aquatic Insect Ecology. I. Biology and Habitat. Wiley, New York. 438 pp.

WARD J.V. \& STANFORD J.A., 1983. The intermediatc disturbance hypothesis: an explanation for biotic diversity patterns in lotic ecosystems. In: T. D. FONTAINE III \& S. M. BARTELL (Editors). Dynamics of lotic ecosystems. Ann Arbor., Michigan, pp. 347-356. 
